\title{
PARTIAL MAPS WITH DOMAIN AND RANGE: EXTENDING SCHEIN'S REPRESENTATION.
}

\author{
MARCEL JACKSON AND TIM STOKES
}

\begin{abstract}
The semigroup of all partial maps on a set under the operation of composition admits a number of operations relating to the domain and range of a partial map. Of particular interest are the operations $R$ and $L$ returning the identity on the domain of a map and on the range of a map respectively. Schein (1970a) gave an axiomatic characterisation of the semigroups with $R$ and $L$ representable as systems of partial maps; the class is a finitely axiomatisable quasivariety closely related to ample semigroups (which were introduced - as type A semigroups - by Fountain, 1979). We provide an account of Schein's result (which until now appears only in Russian) and extend Schein's method to include the binary operations of intersection, of greatest common range restriction, and some unary operations relating to the set of fixed points of a partial map. Unlike the case of semigroups with $R$ and $L$, a number of the possibilities can be equationally axiomatised.
\end{abstract}

\section{INTRODUCTION}

The most ambitious attempt to characterise the algebraic properties of the set of binary relations on a set was initiated by Tarski; this is by now a well-developed part of universal algebra and algebraic logic. Tarski defined his variety of relation algebras by a collection of basic identities concerning intersection, union, complementation, inverse, composition, and constants representing the universal relation, the identity relation and the empty relation. The variety generated by the concrete algebras of all relations on a set turns out to coincide with the class of subdirect products of concrete relation algebras, but is a very complicated subvariety of that initially described by Tarski: for example, the membership problem for the class of finite representable relation algebras is undecidable, see Hirsch and Hodkinson (2001). Both Tarski's relation algebras and the variety generated by the concrete relation algebras remain of considerable interest.

General binary relations have very many natural operations definable on them: this is what makes relation algebras so rich. Restricting attention to specific kinds of relations generally necessitates a restriction in the fundamental operations considered. Thus if one restricts to binary relations that are (total) functions, the only surviving fundamental operations from Tarski's relation algebras are the binary operation of composition and the two nullary operations corresponding to the empty relation and the identity relation. In this way, one obtains the theory of semigroups with some combination of 0 and 1 ; these of course can all be represented in terms of functions on a set. If one restricts to bijective functions, inversion is restored as an

2000 Mathematics Subject Classification. 20M30, 08A02.

Key words and phrases. partial maps, semigroups of functions, closure semigroup, interior semigroup, functional representations.

The first author was supported by ARC Discovery Project Grant DP0342459. 
operation (and 0 is lost) and one obtains the theory of groups. Cayley's Theorem guarantees all abstract groups are representable.

Rather less has been done with (or at least, rather less is well-known about) the set of partial functions $\mathcal{P}(X)$ on a set $X$. We here give a brief overview of some of the relevant possibilities, but the reader should consult the survey by B. Schein (1970b) for a much more detailed treatment. A further survey by Schein and Trokhimenko (1979) concerns algebras of multiplace functions (functions from $X^{n} \rightarrow X$ for some set $\left.X\right)$. A survey article by Jackson and Stokes (2007?) gives some more recent references as well as a more detailed introduction to some of the operations considered below.

Perhaps the most familiar case of algebras representable in $\mathcal{P}(X)$ (aside from semigroups) is the further restricted class of injective partial functions, where inversion as well as composition is well-defined. The axiomatic objects in this case are inverse semigroups, which have received much attention from algebraists for many years; the Vagner-Preston Representation ensures that all abstract inverse semigroups are representable.

The most fundamental question to ask about $\mathcal{P}(X)$ is whether there are any interesting operations (or perhaps term operations) other than composition, $\circ$, that survive from relation algebras; presumably such operations will apply just as well to injective partial maps and so may be related to inverse semigroup operations. One obvious feature of a partial map is that its domain may be a proper restriction of the underlying set. So, in an inverse semigroup, the idempotent element $a^{-1} a$ is represented in the Vagner-Preston theorem as the restriction of the identity to the domain of the partial map corresponding to $a$ (we consider our partial maps as acting on the left). This restriction of the identity $R(f)$ is well-defined for any $f \in \mathcal{P}(X)$.

Semigroups of partial maps with $R$ are closely related to a number of popular "nonregular" generalisations of inverse semigroups. For one example, the weakly right ample semigroups - as studied in Gomes and Gould (1999) for example - are known to correspond to algebras of partial maps with $R$ for which all idempotent elements are restrictions of the identity map. The right type-A semigroups (also known as right ample semigroups) of Fountain (1991) are a similar subclass of the class of all (isomorphic copies of) semigroups of partial maps with $R$. (Further connections are explored below and in the authors' articles, 2001, 2007?, for example.)

Computer scientists have also recently begun to study this operation in the setting of "guarded semigroups" (see Manes, 2006, and also the category theoretic formulation of Cockett and Lack, 2002). It is worth mentioning here that the operation $R$ has interest on the algebra of binary relations on a set, and again computer scientists have re-discovered this operation and its left-sided analog in the algebra of relations, in the setting of "Kleene algebras with domain" (see Desharnais, Möller and Struth, 2006). Such algebras are special cases of "Kleene algebra with tests" (see Kozen, 2000, for example), in which one has a distinguished semilattice of tests (corresponding to restrictions of the identity map). The existence of such a distinguished semilattice is a feature of the current work.

So in this paper we are going to consider the operation $R$, but also several other operations related to the domain and range of a partial map. Conveniently, many of these operations can be described in terms of the restrictions of the identity map id, or equivalently the elements of the form $R(f)$ for some $f \in \mathcal{P}(X)$. For notational 
convenience we define

$$
\Delta(X):=\{e \in \mathcal{P}(X) \mid e \subseteq \mathrm{id}\}
$$

As an example, consider the containment relation $\subseteq$, which partially orders $\mathcal{P}(X)$ (viewing a partial map $f: X \rightarrow X$ as a binary relation $f \subseteq X \times X$ ). This important relation (the "fundamental order") was studied and characterised by Schein (1964) (see also Schein, 1979). When $R$ is present, the fundamental order is trivially seen to coincide with the relation $\leq_{R}$ defined $^{1}$ by $f \leq_{R} g$ if and only if $f=g \circ R(f)$. Equivalently, $f \leq_{R} g$ if and only if $(\exists e \in \Delta(X)) f=g \circ e$.

There are two further domain-related operations we consider.

- The unary operation $I$. For a partial map $f \in \mathcal{P}(X)$ let $I(f)$ denote the restriction of the identity to the points fix $(f)$ fixed by $f$. Equivalently, one may observe

$$
I(f)=\max \{e \in \Delta(X) \mid f \circ e=e\} .
$$

The operation $I$ plays an important role in the inverse algebras of Leech (1995) (where it is equivalent to the presence of the operation of intersection) and is also briefly investigated in Jackson and Stokes (2003). Semigroups with an operation modelling $I$ are investigated by Kelarev and Stokes (1999).

- The binary operation $\wedge$. The binary operation of intersection is also welldefined on $\mathcal{P}(X)$ (considered as a set of binary relations). This can be seen to coincide with the binary operation $\wedge$ defined by

$$
f \wedge g=\max \{z \mid(\exists e \in \Delta(X)) z=f \circ e=g \circ e\} ;
$$

in other words, the largest common domain restriction of $f$ and $g$. For another equivalent definition, note that $f \wedge g$ is equal to the infimum of $\{f, g\}$ under the order $\leq_{R}$, that is $f \wedge g=\max \left\{z \mid z \leq_{R} f\right.$ and $\left.z \leq_{R} g\right\}$. The subalgebras of $\langle\mathcal{P}(X) ; \circ, \wedge\rangle$ are characterised by Garvac'kiu (1971). Subalgebras of $\langle\mathcal{P}(X) ; \circ, \wedge, R\rangle$ are characterised by Dudek and Trokhimenko (2002) and the authors (2003). The class is a variety, and its equational theory is algorithmically described by the authors (2006a).

Each of the "domain operations" $R, I$ and $\wedge$ have corresponding dual operations defined in terms of the range of a map.

- The unary operation $L$. For a partial map $f \in \mathcal{P}(X)$ let $L(f)$ denote the restriction of the identity map to the range of $f$. Equivalently,

$$
L(f)=\min \{e \in \Delta(X) \mid e \circ f=f\} .
$$

The operation $L$ defines a partial order $\leq_{L}$ that is finer than $\leq_{R}$. We write $f \leq_{L} g$ if $f=L(f) \circ g$, or equivalently if $f=L(x) \circ g$ for some $x$; this is routinely seen to be a partial order (for an example, this is Proposition 1.5 of Jackson and Stokes, 2001) that coincides with $\leq_{R}$ on its restriction to $\Delta(X)$. The operation $L$ (in conjunction with $R$ ) was introduced in the series of papers by Schweizer and Sklar $(1960,1961,1965,1967)$. The characterisation of semigroups of partial maps with $R$ and $L$ was established by Schein (1970a), a result of central importance to this article.

\footnotetext{
${ }^{1}$ In this article, partial maps act on the left of a set.
} 
- The unary operation $J$. For a partial map $f \in \mathcal{P}(X)$ let $J(f)$ denote the restriction of the identity to the points fixed by the multi-map $f^{-1}$. Equivalently, one may observe that

$$
J(f)=\max \{e \in \Delta(X) \mid e \circ f=e\},
$$

hence $J$ is just the left sided dual of the operation $I$. For injective maps, the operation $J$ coincides with $I$.

- The binary operation $\curlywedge$. For partial maps $f, g \in \mathcal{P}(X)$ let $f \curlywedge g$ denote the largest common range restriction of $f$ and $g$, that is

$$
f \curlywedge g=\max \{z \mid(\exists e \in \Delta(X)) z=e \circ f=e \circ g\} .
$$

Equivalently, $f \curlywedge g$ denotes the infimum of $\{f, g\}$ under the order $\leq_{L}$. This operation does not seem to have appeared in the literature, but in conjunction with $L$, it is covered by the investigations of Jackson and Stokes (2003). When applied to injective maps, the operation $\curlywedge$ coincides with $\wedge$.

We often refer to these latter operations as range operations.

In this paper, we say that a semigroup with extra operations is representable if it is isomorphic to a subalgebra of some $\mathcal{P}(X)$ endowed with some subset $\Lambda$ of the set of operations $\{R, L, I, J, \wedge, \curlywedge\}$ just described (possibly also including the constants 1 and/or 0 , corresponding to id and $\varnothing$ respectively). Abstract characterisations of representable semigroups with extra operations are known for $\Lambda$ equal to any of the cases $\{R\},\{R, L\},\{\wedge\},\{R, \wedge\}=\{R, I, \wedge\}$ (the equality follows because $I(f)=f \wedge R(f)$ on $\mathcal{P}(X))$; details are given as they arise. The proof in the case $\Lambda=\{R, L\}$ appears to be more difficult than the other cases and was established by Schein (1970a). In this paper we give what seems to be the first English presentation of Schein's elegant construction, and then show how it can be made to yield characterisations and representations for any further subset $\Lambda$ of $\{R, L, I, J, \wedge, \curlywedge, 0,1\}$ satisfying

- $R \in \Lambda$, and

- if $\Lambda$ contains a range operation, then $L \in \Lambda$.

All of the described classes are finitely axiomatisable quasivarieties. We finish by showing which of these are varieties (that is, equationally defined classes): in the case when a range operation is present, this property turns out to be equivalent to when one of $\wedge$ or $\curlywedge$ is present in $\Lambda$.

We stress here that the domain and range operations described above do not exhaust the list of all partial map related operations and relations studied in the literature. For example, the principal objects of investigation in Schein's article (1970a) are the so-called 1-stacks, which have (in addition to composition) two binary operations closely related to $R$ and $L$. Another example is the operation of set subtraction. This is clearly defined for partial maps but is routinely verified not to be expressible as a term in the operations we defined above (we omit the easy proof). Algebras of partial maps with set subtraction and composition were characterised by Schein (1992). Nevertheless, the operations studied in this article constitute the most enriched algebra of partial maps with both domain and range information that have been characterised to date: the 1-stack operations are term functions in $\{\cdot, R, L\}$ (see Section 4 ); while there are currently no published results concerning set subtraction for partial maps in conjunction with either domain- or 
range-related operations. A more detailed analysis of these interrelationships and a fuller overview of known results is given in Jackson and Stokes (2007?).

The content of this paper is as follows. We begin in Section 2 by presenting a general abstraction of the operations defined above. These form a useful "first approximation" to the representable algebras, providing us with many necessary axioms. In Section 3 we characterise the representable semigroups with $\Lambda$ where $\{R\} \subseteq \Lambda \subseteq\{R, I, \wedge, 0,1\}$. We then move on to the case where both $R$ and $L$ are contained in $\Lambda$. In order to keep the work self-contained, in Sections 4 and 5 we give a presentation of Schein's results for semigroups with $R$ and $L$. Our presentation is slightly amplified to help us prove the results in Sections 7 and 8, where Schein's method is shown to extend to any combination of the other operations mentioned above. Finally, in Section 9, we investigate for which combinations of these operations the corresponding class of representable algebras forms a variety.

\section{Preliminaries: a first approximation.}

We begin by establishing some notation that is useful throughout. In the introduction we introduced six operations on the set $\mathcal{P}(X): R, L, I, J, \wedge, \curlywedge$. Each of these operations was described in terms of the subsemilattice on $\Delta(X)$, with the operations $R, L, I, J$ fixing the elements of this. A useful first step toward characterising the subalgebras of reducts of $\langle\mathcal{P} ; \circ, R, L, I, J, \wedge, \curlywedge\rangle$ is to abstract the definitions of these operations in terms of subsemilattices of semigroups.

Let $\Lambda$ be some non-empty subset of $\{R, L, I, J\}$ and let $\mathbf{S}$ be a semigroup with a subsemilattice $\mathbf{E}$ and with each symbol in $\Lambda$ representing a unary operation of $\mathbf{S}$. We say that $\mathbf{S}$ is a $\Lambda$-semigroup with respect to $\mathbf{E}$ if following equalities hold whenever they make sense:

(i) $R(x)=\min \{e \in E \mid x e=x\}$ (right closure);

(ii) $L(x)=\min \{e \in E \mid e x=x\}$ (left closure);

(iii) $I(x)=\max \{e \in E \mid x e=e\}$ (right interior);

(iv) $J(x)=\max \{e \in E \mid e x=e\}$ (left interior).

Evidently, we have $R(S)=L(S)=I(S)=J(S)=E$ (whenever these make sense) and so we need make no reference to $\mathbf{E}$. The class of $\Lambda$-semigroups is a variety, as we now recall.

The $\{R\}$-semigroups first appear in the work of Batbedat (1981) (as type $S L-\gamma$ semigroups) and then the work of Lawson (1991) (as right E-semiadequate semigroups). They are heavily investigated in Jackson and Stokes (2001) where they are given the name $R C$-semigroups (right closure semigroups). The class of RCsemigroups is the variety of unary semigroups satisfying

(R1) $x R(x) \approx x$

(R2) $R(x) R(y) \approx R(y) R(x)$,

(R3) $R(R(x)) \approx R(x)$, and

(R4) $R(x y) R(y) \approx R(x y)$.

The class of $\{L\}$-semigroups is axiomatised dually (and we denote the corresponding axioms by (L1)-(L4) respectively). These are called LC-semigroups in Jackson and Stokes (2001). The class of $\{I\}$-semigroups is characterised in Kelarev and Stokes (1999) under the name interior semigroups. It is shown that interior semigroups form the variety of unary semigroups satisfying

(I1) $I(I(x)) \approx I(x)$, 
(I2) $x I(x) \approx x$,

(I3) $I(x) I(y) \approx I(y) I(x)$,

(I4) $I(x y) I(y) \approx I(x) I(y)$.

The class of $\{J\}$-semigroups is axiomatised dually (and we denote the corresponding axioms by (J1)-(J4) respectively).

These individual cases can be combined as follows.

Lemma 2.1. Let $\Lambda$ be a subset of $\{R, L, I, J\}$. The class of all $\Lambda$-semigroups is a variety defined by associativity of $\cdot$ and the axioms $(G 1)-\left(G_{4}\right)$ for each $G \in \Lambda$ and $G(H(x)) \approx H(x)$ for each $G, H \in \Lambda$.

Proof. The proof is obvious in view of the individual axiomatisations and is omitted.

In particular, as $\langle\mathcal{P}(X) ; \circ, \Lambda\rangle$ is a $\Lambda$-semigroup (with respect to $\Delta(X)$ ), so are all algebras representable in it.

Every subsemilattice $\mathbf{E}$ of a semigroup $\mathbf{S}$ determines two partial orders extending

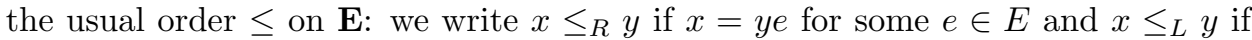
$x=e y$ for some $e \in E$. It is desirable to define a generalisation of the operations $\wedge$ and $\curlywedge$, which on $\mathcal{P}(X)$ can be defined in terms of these orders relative to $\Delta(X)$. Accordingly, if $\Lambda$ is a non-empty subset of $\{R, L, I, J, \wedge, \curlywedge, 0,1\}$ then a semigroup with extra operations in $\Lambda$ (where $\Lambda$ and $\curlywedge$ are binary operations) is a $\Lambda$-semigroup with respect to a subsemilattice $\mathbf{E}$ if in addition to the previously stated properties we have

(v) $x \wedge y=\inf \{x, y\}$ (right meet) under the order on $\left\langle S ; \leq_{R}\right\rangle$;

(vi) $x \curlywedge y=\inf \{x, y\}$ (left meet) under the order on $\left\langle S ; \leq_{L}\right\rangle$,

(vii) 0 is the zero element for $\mathbf{S}$ and $0 \in E$,

(viii) 1 is the identity element for $\mathbf{S}$ and $1 \in E$.

Again, $\mathcal{P}(X)$ becomes a $\Lambda$-semigroup; however, the definition is not particularly sensible unless we ask for an additional property to be satisfied. We say that $\Lambda$ is well-formed if it satisfies $\wedge \in \Lambda \Rightarrow R \in \Lambda$ and $\curlywedge \in \Lambda \Rightarrow L \in \Lambda$.

To see what is wrong with subsets $\Lambda \subseteq\{R, L, I, J, \wedge, \curlywedge, 0,1\}$ that are not wellformed, observe that on $\langle\mathcal{P}(X) ; \circ, \Lambda\rangle$ the operations $\wedge$ and/or $\curlywedge$ are defined in terms of the subsemilattice $\Delta(X)$, however it is not hard to find subalgebras of $\langle\mathcal{P}(X) ; \circ, \Lambda\rangle$ where the corresponding restriction of $\wedge$ and/or $\curlywedge$ are not defined in terms of any subsemilattice. Hence in this case, the class of $\Lambda$-semigroups does not include the desired representable algebras. This problem is rectified when $\Lambda$ is well-formed because the orders $\leq_{R}$ and $\leq_{L}$ are more precisely captured in terms of the operations (. and) $R$ and $L$, respectively: $x \leq_{R} y$ if and only if $x=y R(x)$ and $x \leq_{L} y$ if and only if $x=L(x) y$ (see Jackson and Stokes, 2001, for example). Thus in the presence of $R$ (or $L$ ), the order $\leq_{R}$ (or $\leq_{L}$ ) simply restricts on taking subalgebras.

Note that $\{R, \wedge\}$-semigroups are also known as semilattice ordered $R C$-semigroups, or $S L O R C s$. The class of SLORCs is shown to be a variety in Jackson and Stokes (2003); the required identities are (R1)-(R4) above, plus the axioms

$(\wedge 1) x \wedge y \approx y \wedge x, x \wedge x \approx x,(x \wedge y) \wedge z \approx x \wedge(y \wedge z)$,

$(\wedge 2)(x \wedge y) R(z) \approx(x R(z) \wedge y R(z)) R(z)$,

$(\wedge 3)(x \wedge y)=x R(x \wedge y)$. 
The class of $\{L, \curlywedge\}$-semigroups (SLOLCs) can be axiomatised dually (and we denote the corresponding axioms $(\curlywedge 1)-(\curlywedge 3)$ respectively). The only identities required to capture the nullaries 0 and 1 are the usual multiplicative properties of these elements plus the laws $G(0) \approx 0$ and $G(1) \approx 1$ for some $G \in\{R, L, I, J\} \cap \Lambda$.

Summarising, we can extend Lemma 2.1 as follows.

Lemma 2.2. Let $\Lambda$ be a well-formed subset of $\{R, L, I, J, \wedge, \curlywedge, 0,1\}$. The class of all $\Lambda$-semigroups is a variety defined by associativity of $\cdot$ and the axioms

- (G1)-(G4) for each $G \in \Lambda \cap\{R, L, I, J\}$,

- $G(H(x)) \approx H(x)$ for each $G, H \in \Lambda \cap\{R, L, I, J\}$,

- $(\perp 1)-(\perp 3)$, for each $\perp \in \Lambda \cap\{\wedge, \curlywedge\}$,

- $x 0 \approx 0 \approx 0 x \approx H(0)$ if $0 \in \Lambda$ and $H \in \Lambda \cap\{R, L, I, J\}$,

- $x 1 \approx x \approx 1 x$ and $H(1) \approx 1$ if $1 \in \Lambda$ and $H \in \Lambda \cap\{R, L, I, J\}$.

In this paper we will adopt the convention that a $\Lambda$-semigroup $\mathbf{S}$ contains an element labeled 0 or 1 if and only if $0 \in \Lambda$ or $1 \in \Lambda$ respectively. Put another way, if 0 (or 1 ) is not in $\Lambda$ then any multiplicative zero element (identity element) of a $\Lambda$-semigroup must be denoted by some symbol other than 0 (or 1 ). Hence $S \backslash\{0\}=S$ if and only if $0 \notin \Lambda$ (and similarly for $S \backslash\{1\}$ ).

The following lemma shows that we can omit mention of $I$ or $J$ in $\Lambda$ if $R, \wedge \in \Lambda$ or if $L, \curlywedge \in \Lambda$, respectively.

Lemma 2.3. The variety of $\{R, I, \wedge\}$-semigroups is term equivalent to that of $\{R, \wedge\}$-semigroups.

Proof. Let $\mathbf{S}$ be a $\{R, \wedge\}$-semigroup. Let $I^{\prime}(x):=x \wedge R(x)$. We show that $I^{\prime}=I$. By the definition of $\wedge$, we find that $I^{\prime}(x)=\max \{z \mid(\exists e=R(e)) z=x e=R(x) e\}$. Now $R(x) e$ is closed, and $I^{\prime}(x)$ satisfies $I^{\prime}(x)=x e=R(x) e$ for some $e$. So $I^{\prime}(x)$ is closed and satisfies $x I^{\prime}(x)=x R(x) e=x e=I^{\prime}(x)$ for some $e$. Conversely, if $x e=e$ for some $e \in R(S)$, then $e=x e=x R(x) e=x e R(x)=e R(x)$, hence $e \leq I^{\prime}(x)$. So $I=I^{\prime}$.

There is a useful alternative approach to $\{R, \wedge\}$-semigroups. Let $x * y$ denote the term $R(x \wedge y)$. Then $R(x)=x * x$ (by axiom $(\wedge 1)$ ) and $x \wedge y=x(x * y)$ (by axiom $(\wedge 3))$, so that the defining axioms of $\{R, \wedge\}$-semigroups can be rewritten solely in terms of - and $*$; the corresponding models are known as (right) agreeable semigroups. The relationship just described between $*$ and $R, \wedge$ is a term equivalence between the variety of SLORCs and the variety of agreeable semigroups; see Jackson and Stokes (2003) for this and many other results relating to $*$.

The operation $*$ admits a natural interpretation on $\mathcal{P}(X)$; namely, $f * g$ is the identity map on the points of agreement of the partial maps $f$ and $g$. One advantage to the agreeable semigroup perspective of SLORCs is that the operation $*$ has similarities with many properties of the relation of equality: for example the identity $x(x * y) \approx y(x * y)$ holds on any agreeable semigroup, which we can think of as saying (very loosely) that

$$
\text { " } x \text { and } x=y \text { " is the same as " } y \text { and } x=y " .
$$

While this connection is weak and imprecise, it is this interpretation that provides the central idea underlying the algorithmic description of the equational theory of representable SLORCs in Jackson and Stokes (2006a).

There are corresponding left-sided versions of agreeable semigroups; in this paper we use the symbol $\star$ for left agreeable operations. The notion of a $\Lambda$-semigroup 
extends in the obvious way to include the operations $*$ and $\star$. Naturally, we do not need to include $\wedge$ or $R$ if $*$ is in $\Lambda$, or $L$, $\curlywedge$ if $\star$ is in $\Lambda$. Lemma 2.3 shows that the terms $I(x)$ and $x *(x * x)$ coincide, as do $J(x)$ and $(x \star x) \star x$.

For $A, B \subseteq\{R, L, I, J, \wedge, \curlywedge, *, \star, 0,1\}$, we write $A \leq B$ if there are $A^{\prime}$ and $B^{\prime}$ which are equivalent modulo the equivalences $\{R, I, \wedge\} \equiv\{R, \wedge\} \equiv\{*\},\{L, J, \curlywedge\} \equiv$ $\{L, \curlywedge\} \equiv\{\star\}$ to $A$ and $B$ respectively and such that $A^{\prime} \subseteq B^{\prime}$.

2.1. Examples. The set $\mathcal{B}(X)$ of all binary relations on a set $X$ (here viewed as multi-valued partial maps acting on the left of $X)$ is a semigroup under composition containing $\langle\mathcal{P}(X) ; \circ\rangle$ as a subsemigroup. The semigroup $\langle\mathcal{B}(X) ; \circ\rangle$ becomes a $\Lambda$ semigroup if the operations in $\Lambda$ are defined in terms of the subsemilattice $\Delta(X)$. Note however that $\wedge$ on $\mathcal{B}(X)$ is no longer equal to $\cap$ : the value of $r_{1} \wedge r_{2}$ is the largest common domain restriction of the binary relations $r_{1}$ and $r_{2}$. The operations $R$ and $L$ on $\mathcal{B}(X)$ (in conjunction with other operations) were studied by Bredihin (1977) and more recently arose in the context of propositional Hoare Logic in the work of Desharnais, Möller and Struth (2006).

As a further example of interest, let $\mathbf{A}$ be any algebraic structure and consider the set $\operatorname{End}(\mathbf{A})$ consisting of all local endomorphisms of $\mathbf{A}$ : partial maps on $\mathbf{A}$ whose domains are subalgebras of $\mathbf{A}$ and which satisfy the homomorphism properties for $\mathbf{A}$. These partial maps are closed under composition, and the subset $E$ of $\operatorname{End}(\mathbf{A})$ consisting of the restrictions of the identity map to subalgebras of $\mathbf{A}$ forms a subsemilattice $\mathbf{E}$ of $\langle\operatorname{End}(\mathbf{A}) ; \circ\rangle$. It is routinely verified that $\langle\operatorname{End}(\mathbf{A}) ; \circ\rangle$ becomes a $\{R, L, \wedge, \curlywedge, 0,1\}$-semigroup relative to $\mathbf{E}$. Moreover, it is a subalgebra of $\langle\mathcal{P}(A) ; \circ, R, L, \wedge, \curlywedge, \varnothing, \mathrm{id}\rangle$ and so is trivially representable. (Note also that $\mathcal{P}(A)$ is itself equal to the set of local endomorphisms of the algebra $\langle A ;\rangle$ with no operations.)

The injective elements of $\operatorname{End}(\mathbf{A})$ - denoted collectively Aut $(\mathbf{A})$ - are often called local automorphisms of $\mathbf{A}$, and form a subuniverse of $\langle\operatorname{End}(\mathbf{A}) ; \circ, R, L, \wedge, \curlywedge, 0,1\rangle$. The operations $\wedge$ and $\curlywedge$ can be seen to coincide on $\operatorname{Aut}(\mathbf{A})$, and if the operation ${ }^{-1}$ of inversion is added, the operations $L$ and $R$ become term operations: $R(x)=$ $x^{-1} \circ x$ and $L(x)=x \circ x^{-1}$. The structures $\left\langle\operatorname{Aut}(\mathbf{A}) ; \circ, \wedge,{ }^{-1}\right\rangle$ are the main object of investigation in Bredihin (1976), and one of the central motivating examples in Leech (1995).

\section{Domain Operations}

The operations $R, I$ and $\wedge$ are defined on $\mathcal{P}(X)$ in terms of domain restrictions, and so we will call them domain operations. In this section we recall some of the known properties of $\Lambda$-semigroups when $\{R\} \leq \Lambda \leq\{R, I, \wedge, 0,1\}$ (such $\Lambda$ are obviously well-formed).

Let $\Lambda$ be a subset of $\{R, I, \wedge, 0,1\}$ containing $R$. The class of representable RC-semigroups seems to have been first explicitly characterised by Trokhimenko (1973); indeed an $n$-ary function version is obtained there. The characterisation was rediscovered by the authors (2001) and then again by Manes (2006). A category theoretic version of the result was proved by Cockett and Lack (2002). For any $\Lambda$ semigroup $\mathbf{S}$ with $R \in \Lambda$ we may define the map $\psi: S \rightarrow \mathcal{P}(S)$ by letting $\psi_{a}$ be the partial map with domain $R(a) S$ and with action $x \mapsto a x$. An RC-semigroup is representable if and only if it satisfies the twisted law:

(R5) $R(x) y \approx y R(x y)$. 
(Note that we do not yet define a left dual law (L5).) More specifically, the map $\psi$ is a faithful representation for $\langle S ; \cdot, R\rangle$ in $\langle\mathcal{P}(S) ; \circ, R\rangle$ (a different representing map is used in Trokhimenko, 1973). An inverse semigroup $\mathbf{S}=\left\langle S ; \cdot,^{-1}\right\rangle$ admits the structure of an RC-semigroup by letting $R(x):=x^{-1} x$, and moreover, this unary operation satisfies (R5). For such $\mathbf{S}$, the map $\psi$ coincides with the Vagner-Preston representation; see Jackson and Stokes (2001) for example.

The map $\psi$ also preserves 1 if it is present, but not 0 . Let us define $\psi^{b}: S \rightarrow$ $\mathcal{P}(S \backslash\{0\})$ by setting $\psi_{a}^{b}$ (for each $a \in S$ ) to be the restriction of $\psi_{a}$ in both its domain and range to the set $S \backslash\{0\}$. The following lemma makes it obvious that this is also a faithful representation, that moreover preserves 0 .

Lemma 3.1. Let $\mathbf{S}$ be a $\Lambda$-semigroup with $\{R, 0\} \subseteq \Lambda$ such that $R$ is twisted. Then for every $x \in S$, the partial map $\psi_{x}$ is the disjoint union of the map $0 \mapsto 0$ with the map $\psi_{x}^{b}$, whose domain and range lies in $S \backslash\{0\}$.

Proof. Certainly every element of $\psi(S)$ fixes the element 0 . Now suppose $\psi_{x}(a)=0$ for some $x, a \in S$. By the definition of $\psi$ we have $R(x) a=a$ and $x a=0$, so using (R5) we get $a=R(x) a=a R(x a)=a R(0)=a 0=0$. So the only point mapping to 0 is 0 itself.

Of course, our convention on 0 means that $\psi^{b}=\psi$ if $0 \notin \Lambda$. We summarise all this by the following.

Theorem 3.2. Let $\{R\} \subseteq \Lambda \subseteq\{R, 0,1\}$. The representable $\Lambda$-semigroups are defined within the variety of $\Lambda$-semigroups by the twistedness of $R$.

In Jackson and Stokes (2003), the map $\psi$ is used to characterise the representable $\{R, \wedge\}$-semigroups: in addition to the twistedness of $R$ (law (R5)), the extra law ( $x \wedge$ $y) z \approx x z \wedge y z$ must be satisfied (a multiplace version of this result was obtained by Dudek and Trokhimenko, 2002, although the representing map is different). These algebras are called twisted SLORCs in Jackson and Stokes (2003). By Lemma 3.1, the map $\psi^{b}$ also preserves $\wedge$.

Theorem 3.3. Let $\{R, \wedge\} \leq \Lambda \leq\{R, \wedge, 0,1\}$. The representable $\Lambda$-semigroups are defined within the variety of $\Lambda$-semigroups by the twistedness of $R$ and the law $(x \wedge y) z \approx x z \wedge y z$.

The corresponding class of agreeable semigroups is the variety of all agreeable semigroups satisfying the law $(x * y) z \approx z(x z * y z)$; this law is the twisted law for (right) agreeable semigroups. The equational theory of twisted agreeable semigroups with or without 0 and 1 is effectively described in Jackson and Stokes (2006a) (and hence an effective algorithm is known for the equational theory of representable $\Lambda$-semigroups with $\Lambda \leq\{*, 0,1\})$.

We now investigate the representable $\{R, I\}$-semigroups with or without 0 or 1 . These do not seem to have been described in the literature, although inverse semigroups with $I$ have been considered, namely in Jackson and Stokes (2003), Kelarev an Stokes (1999) and Leech (1995) (where $I$ is called the fixed point operator).

Theorem 3.4. Let $\{R, I\} \leq \Lambda \leq\{R, I, 0,1\}$. A $\Lambda$-semigroup $\mathbf{S}=\langle S ; \cdot, \Lambda\rangle$ is representable if and only if it is representable as an $\{R\}$-semigroup (so the twisted law (R5) holds) and the quasi-identity

(I5) $x y \approx y \rightarrow I(x) y \approx y$ 
is satisfied.

Proof. Certainly $\mathbf{S}$ must be representable as an $\{R\}$-semigroup. Verification of the quasi-identity (I5) on $\langle\mathcal{P}(X) ; \circ, R, I\rangle$ is routine and left to the reader. Thus it suffices to show that the twisted $\{R, I\}$-semigroup $\mathbf{S}$ is representable if it satisfies (I5). We show that the RC-semigroup representation $\psi^{b}$ preserves $I$.

Now as $\psi^{b}$ preserves $R$ we have $R\left(\psi_{I(x)}^{b}\right)=\psi_{R(I(x))}^{b}=\psi_{I(x)}^{b}$ and so $\psi_{I(x)}^{b}$ is closed. Also, as $\psi_{x}^{b} \circ \psi_{I(x)}^{b}=\psi_{x I(x)}^{b}=\psi_{I(x)}^{b}$, we find that $\psi_{I(x)}^{b} \leq_{R} I\left(\psi_{x}^{b}\right)$. Now suppose $a \in \operatorname{dom}\left(I\left(\psi_{x}^{b}\right)\right)$. This implies $\psi_{x}^{b}(a)=a \neq 0$ and hence $x a=a$ in S. By (I5), we can deduce that $I(x) a=a$. But then $R(I(x)) a=I(x) a=a$ so that $a \in \operatorname{dom}\left(\psi_{I(x)}^{b}\right)$ as required.

We also observe here that Garvac'kiu (1971) characterised the algebras $\langle S ; \cdot, \wedge\rangle$ representable in $\langle\mathcal{P}(X) ; \circ, \wedge\rangle$ (as explained above, these are not $\{\wedge\}$-semigroups). This class is a variety: in addition to associativity of $\cdot$ and the usual semilattice laws for $\wedge$, the required identities are $(x \wedge y) z \approx x z \wedge y z$ and $x(a \wedge b \wedge c) \wedge y(b \wedge c) \approx$ $x(a \wedge c) \wedge y(b \wedge c)$.

\section{FUnCTION SYSTEMS}

We now turn to $\Lambda$-semigroups when both $R$ and $L$ are contained in $\Lambda$. The situation is much more complicated than in the domain-only case.

Following early efforts by Menger (1959), variations of $\{R, L\}$-semigroups (sometimes with 1) were investigated in a series of papers by Schweizer and Sklar (1960, $1961,1965,1967)$, with a view to characterising various properties of $\langle\mathcal{P}(X) ; R, L\rangle$. Their approach culminated in the notion of a type 2 function system, which one can show is equivalent to an $\{R, L\}$-semigroup in which $R$ is twisted and $L$ satisfies the law

(L5) $L(x y) \approx L(x L(y))$.

(See the survey article Jackson and Stokes, 2007?, for a fuller exposition of this connection.) Following Jackson and Stokes (2001), we call (L5) the left congruence condition (it is equivalent to the kernel of $L$ being a left congruence; see Jackson and Stokes, 2001). Note that (L5) is not the left dual of (R5).

It is easy to verify that the $\{R, L\}$-semigroup $\langle\mathcal{P}(X) ; \circ, R, L\rangle$ is a type 2 function system. In Schweizer and Sklar (1967), a "proof" that any type 2 function system can be embedded in $\langle\mathcal{P}(X), R, L\rangle$ is given; this would characterise the representable $\{R, L\}$-semigroups. However, Schein proved that the additional quasi-identity

(L6) $x z \approx y z \rightarrow x L(z) \approx y L(z)$

is required, and that this quasi-identity cannot be replaced by any equivalent set of identities Schein (1970a) (see also Schein, 1970b). Hence the representable $\{R, L\}$ semigroups are a proper quasivariety. ${ }^{2}$

It is possible to show that the map described by Schweizer and Sklar is the same as the map $\psi$ used to represent RC-semigroups in Jackson and Stokes (2001). (This is not quite immediate, and by itself does not yield the RC-semigroup part of Theorem 3.2 since the operation $L$ plays a central role in both the description

\footnotetext{
${ }^{2}$ We mention here that the class of $\{L, R\}$-semigroups satisfying right twistedness of $R$, left twistedness of $L$ (the left dual of law (R6) for $L$ ), law (L6) and its right dual for $R$ have received substantial attention in the literature: they are precisely the ample semigroups of Fountain, 1979 (there they are called type A semigroups, but later renamed as ample semigroups).
} 
and proof.) However, even with the extra axiom (L6) at hand, $\psi$ is insufficient to provide the desired representation: there are representable $\{R, L\}$-semigroups $\mathbf{S}$ for which $\psi_{L(x)}$ is strictly larger than $L\left(\psi_{x}\right)$ for some $x \in S$. Instead, Schein devised a much more elaborate method which attempts to represent an $\{R, L\}$ semigroup $\mathbf{S}$ over special sequences of elements of $S$. In this section we will give a description of this construction. The proof that the described set embedding preserves the various operations we wish to consider is left to sections to follow. Our presentation is occasionally more detailed than that of Schein, but all of the results in this section are essentially contained in Schein (1970a), or by modification of results in Schein (1970a).

If $\mathbf{A}=\langle A ; \cdot R, L\rangle$ is a type 2 function system, additionally satisfying Schein's implication (L6), we here call it a type 3 function system. Schein's representation for type 3 function systems is indirect in that his primary algebraic objects are stacks, in which the fundamental operations are composition, and two binaries $\triangleleft$ and $\triangleright$. Any type 2 function system (hence, any type 3 function system) can be viewed as a stack where we define $a \triangleright b:=b R(a)$ and $a \triangleleft b:=L(b) a$, however the operations $R$ and $L$ are not definable on every stack and hence the type 3 function systems have slightly greater expressive power than the class of stacks. Schein obtains a representation for type 3 function systems via a modification of his corresponding representation for stacks (also given in Schein, 1970a) . $^{3}$

Throughout, let $\mathbf{A}=\langle A ; \cdot, R, L\rangle$ be a type 3 function system.

Lemma 4.1. For $a, b, c, d \in A$, suppose $a c=b c$ and $L(c)=L(d)$. Then $a d=b d$.

Proof. If $a c=b c$ then $a L(c)=b L(c)$, so $a L(d)=b L(d)$, and so post-multiplying by $d$ gives $a d=b d$.

We call this the replation rule (replacement on cancellation). A simple modification applies to cases of the form $a c=c$.

Let $\mathbf{A}=\langle A ; \cdot, R, L\rangle$ be a type 3 function system. By a word over $A$ we mean simply an element of the free semigroup over the alphabet $A$. We use the notation $\left(a_{1}, a_{2}, \ldots, a_{n}\right)$ in preference to the usual $a_{1} a_{2} \ldots a_{n}$ because the latter is indistinguishable from a product in $\mathbf{A}$; we also allow notation such as $\left(a_{1}, a_{2}, \ldots\right)$ when the values of $n$ and $a_{n}$ are not important. A word $\left(a_{0}, b_{1}, a_{1}, \ldots, b_{n}, a_{n}\right)$ (of odd length) is permissible if $L\left(a_{i}\right)=L\left(b_{i}\right)$ and $R\left(a_{i}\right)=R\left(b_{i+1}\right)$ for all $i$ for which this makes sense. Let $\mathrm{p}(A)$ denote the set of all permissible words over $A$.

Lemma 4.2. If $\mathbf{A}$ is in addition a $\{R, L, 0\}$-semigroup $($ so $R(0)=0)$ then the only permissible words containing 0 are the constant words $(0,0, \ldots, 0)$.

Proof. This is because the implications $R(x) \approx 0 \rightarrow x \approx 0$ and $L(x) \approx 0 \rightarrow x \approx 0$ are obvious consequences of laws (R1) and (L1).

For $\alpha=\left(a_{0}, b_{1}, a_{1}, \ldots, b_{n}, a_{n}\right) \in \mathrm{p}(A)$ and $f \in A$, we say that $\alpha \lessdot f$ if and only if $R(f) a_{0}=a_{0}$. In that case, also define $f \alpha=\left(f a_{0}, b_{1}, a_{1}, \ldots, b_{n}, a_{n}\right)$ (here $f a_{0}$ is of course the product of $f$ with $a_{0}$ in $\mathbf{A}$ ), which is also permissible since $R\left(f a_{0}\right)=R\left(R(f) a_{0}\right)=R\left(a_{0}\right)$.

\footnotetext{
${ }^{3}$ Schein's representation for stacks is in terms of partial maps acting on the right, while we (and Schweizer and Sklar) are representing partial maps acting on the left of a set. Obvious modifications of axioms and definitions allow one to translate between these two possibilities.
} 
We are going to define a notion of reduction for permissible words. If

$$
\alpha:=\left(a_{0}, b_{1}, a_{1}, \ldots, b_{n}, a_{n}\right) \in \mathrm{p}(A)
$$

and $a_{0}=x b_{1}$ (where $x \in A$ or is empty), then reduce $\alpha$ to $\left(x a_{1}, b_{2}, a_{2}, \ldots, b_{n}, a_{n}\right)$; the result is still permissible, as is easily checked.

Lemma 4.3. Reduction of permissible words over $A$, if applicable, is unique.

Proof. Let $\left(a_{0}, b_{1}, a_{1}, \ldots, b_{n}, a_{n}\right) \in \mathrm{p}(A)$. Suppose $a_{0}=x b_{1}$ and $a_{0}=y b_{1}$. Then $x b_{1}=y b_{1}$ so by the replation rule we get $x a_{1}=y a_{1}$.

A word over $A$ is reduced if it cannot be reduced by the above method of reduction. Let the set of all reduced permissible words over $A$ be denoted by $W(A)$. By Lemma 4.3, each $\alpha \in \mathrm{p}(A)$ has a unique reduced form in $W(A)$, denoted by $\bar{\alpha}$.

Lemma 4.4. For any $\alpha \in \mathrm{p}(A)$ and $f \in A$ we have $\alpha \lessdot f$ if and only if $\bar{\alpha} \lessdot f$.

Proof. Let $\alpha=\left(a_{0}, b_{1}, a_{1}, \ldots\right) \in \mathrm{p}(A)$ and assume that $\alpha$ can be reduced to $\beta=$ $\left(x_{1} a_{1}, b_{2}, \ldots\right) \in W(A)$ (where $\left.a_{0}=x_{1} b_{1}\right)$. Observe that $L\left(x_{1} a_{1}\right)=L\left(x_{1} L\left(a_{1}\right)\right)=$ $L\left(x_{1} L\left(b_{1}\right)\right)=L\left(x_{1} b_{1}\right)=L\left(a_{0}\right)$. Hence $\alpha \lessdot f$ if and only if $R(f) a_{0}=a_{0}$ if and only if $R(f) \geq L\left(a_{0}\right)$ if and only if $R(f) \geq L\left(x_{1} a_{1}\right)$ if and only if $\beta \lessdot f$. Hence we also have $\alpha \lessdot f$ if and only if $\bar{\alpha} \lessdot f$.

Lemma 4.5. The only permissible words reducing to the word (0) (if 0 is present), are the words $(0, \ldots, 0)$.

Proof. We have $(0) \lessdot 0$ while $\left(a_{0}, b_{1}, a_{1}, \ldots\right) \lessdot 0$ implies $a_{0}=R(0) a_{0}=0 a_{0}=0$, showing that $a_{0}=b_{1}=\ldots=0$ by Lemma 4.2 .

Lemma 4.6. For any $\alpha \in \mathrm{p}(A)$ and any $g, h \in A$ for which $\alpha \lessdot g$ and $\alpha \lessdot h$ we have $g \alpha=h \alpha$ if and only if $\overline{g \alpha}=\overline{h \alpha}$.

Proof. One direction is obvious. For the other, let $\alpha=\left(a_{0}, b_{1}, a_{1}, \ldots, b_{n}, a_{n}\right) \in$ $\mathrm{p}(A)$. Suppose $\overline{g \alpha}=\overline{h \alpha}=\left(x_{k} a_{k}, b_{k+1}, a_{k+1}, \ldots, b_{n}, a_{n}\right)$, so that

$$
\begin{aligned}
g a_{0} & =x_{1} b_{1} \\
x_{1} a_{1} & =x_{2} b_{2} \\
& \vdots \\
x_{k-1} a_{k-1} & =x_{k} b_{k} .
\end{aligned}
$$

and

$$
\begin{aligned}
h a_{0} & =y_{1} b_{1} \\
y_{1} a_{1} & =y_{2} b_{2} \\
& \vdots \\
y_{k-1} a_{k-1} & =y_{k} b_{k}
\end{aligned}
$$

and $x_{k} a_{k}=y_{k} a_{k}$. So $x_{k} b_{k}=y_{k} b_{k}$ by replation, so $x_{k-1} a_{k-1}=y_{k-1} a_{k-1}$, and repeating we obtain that $x_{1} b_{1}=y_{1} b_{1}$, so that $g a_{0}=h a_{0}$, and so $g \alpha=h \alpha$.

Lemma 4.7. For any $\alpha \in \mathrm{p}(A)$ and any $f \in A$ for which $\alpha \lessdot f, \overline{f \alpha}=\overline{f \bar{\alpha}}$. 
Proof. By Lemma 4.4, it suffices to show that $f \alpha$ can be reduced to $f \bar{\alpha}$; while further reductions may still be possible, the reduced form will be the same for both.

If $\alpha=\left(a_{0}, b_{1}, a_{1}, \ldots, b_{n}, a_{n}\right) \in \mathrm{p}(A)$, suppose $\bar{\alpha}=\left(x_{k} a_{k}, b_{k+1}, a_{k+1}, \ldots, b_{n}, a_{n}\right) \in$ $W(A)$. Hence $a_{0}=x_{1} b_{1}, x_{1} a_{1}=x_{2} b_{2}, \ldots, x_{k-1} a_{k-1}=x_{k} b_{k}$, so multiplying on the left by $f$ gives $f a_{0}=f x_{1} b_{1}, f x_{1} a_{1}=f x_{2} b_{2}, \ldots, f x_{k-1} a_{k-1}=f x_{k} b_{k}$. Uniqueness of reductions means that this sequence of reductions applies also to $f \alpha$. Hence $f \alpha$ reduces to $f \bar{\alpha}$, as required.

Corollary 4.8. For any $\alpha \in \mathrm{p}(A)$ and $g, h \in A, \overline{g \bar{\alpha}}=\overline{h \bar{\alpha}}$ if and only if $g \alpha=h \alpha$.

Proof. $\overline{g \bar{\alpha}}=\overline{g \alpha}$ and $\overline{h \bar{\alpha}}=\overline{h \alpha}$ by the previous lemma; now apply Lemma 4.6.

Corollary 4.9. For any $\alpha \in \mathrm{p}(A)$ and any $g, h \in A, g \alpha=h \alpha$ if and only if $g \bar{\alpha}=h \bar{\alpha}$.

Proof. $g \alpha=h \alpha$ if and only if $\overline{g \alpha}=\overline{h \alpha}$ if and only if $\overline{g \bar{\alpha}}=\overline{h \bar{\alpha}}$ if and only if $g \bar{\alpha}=h \bar{\alpha}$.

Corollary 4.10. For any $\alpha \in \mathrm{p}(A)$ and any $g \in A, g \alpha=\alpha$ if and only if $g \bar{\alpha}=\bar{\alpha}$.

Proof. If $\alpha=\left(a_{0}, \ldots\right) \in \mathrm{p}(A)$, then $\alpha=L\left(a_{0}\right) \alpha$ and then $L\left(a_{0}\right) \bar{\alpha}=\bar{\alpha}$ by Lemma 4.4. Now apply Corollary 4.9 to $g \alpha=L\left(a_{0}\right) \alpha$.

This completes the required information concerning permissible and reduced words. For any type 3 function system $A$, Schein defines the following map. For each $g \in A$, define $\xi_{g}: W(A) \rightarrow W(A)$ with $\operatorname{dom}\left(\xi_{g}\right)=\{\alpha \in W(A): \alpha \lessdot g\}$ and action $\alpha \mapsto \overline{g \alpha}$. We similarly define $\xi^{b}$ by letting $\xi_{g}^{b}$ be the restriction of $\xi_{g}$ in its domain and range to $W(A) \backslash\{(0)\}$.

\section{Preservation of $R, L$ And .}

We now continue our exposition of Schein's representation by showing that the map $g \mapsto \xi_{g}$ is an embedding of type 3 function systems.

Theorem 5.1. For all $g, h \in A, \xi_{h} \circ \xi_{g}=\xi_{h g}$.

Proof. Suppose $\alpha=\left(a_{0}, b_{1}, a_{1}, \ldots\right) \in W(A)$.

First suppose that $\alpha \in \operatorname{dom}\left(\xi_{h} \circ \xi_{g}\right)$, so that $\alpha \lessdot g$ and $\xi_{g}(\alpha) \lessdot h$, that is, $\overline{g \alpha} \lessdot h$. Then $R(g) \alpha=\alpha$ and $R(h) \overline{g \alpha}=\overline{g \alpha}$. Then $R(h) g \alpha=g \alpha$ by Lemma 4.6. Writing $\alpha=\left(a_{0}, b_{1}, a_{1}, \ldots\right)$, these facts say that $R(g) a_{0}=a_{0}$ and $R(h) g a_{0}=g a_{0}$. Hence $R(h g) a_{0}=R(R(h) g) a_{0}=a_{0} R\left(R(h) g a_{0}\right)=a_{0} R\left(g a_{0}\right)=R(g) a_{0}=a_{0}$, and so $R(h g) \alpha=\alpha$, so $\alpha \lessdot h g$, or $\alpha \in \operatorname{dom}\left(\xi_{h g}\right)$.

Now for such $\alpha, \xi_{h}\left(\xi_{g}(\alpha)\right)=\overline{h \overline{g \alpha}}=\overline{h(g \alpha)}=\overline{(h g) \alpha}=\xi_{h g}(\alpha)$, by Lemma 4.7 and using the definition of $f \alpha$ where $\alpha \lessdot f$.

It remains to show that if $\alpha \in \operatorname{dom}\left(\xi_{h g}\right)$ then $\alpha \in \operatorname{dom}\left(\xi_{h} \circ \xi_{q}\right)$. Now suppose $\alpha \lessdot h g$, that is, $R(h g) a_{0}=a_{0}$. Then $R(g) a_{0}=R(g) R(h g) a_{0}=R(h g) a_{0}=a_{0}$, and so $\alpha \lessdot g$. Also, $R(h) g a_{0}=g R(h g) a_{0}=g a_{0}$, so $R(h) g \alpha=g \alpha$, and so $R(h) \overline{g \alpha}=\overline{g \alpha}$, so $\overline{g \alpha} \lessdot h$.

Theorem 5.2. For $g \in A, R\left(\xi_{g}\right)=\xi_{R(g)}$.

Proof. Clearly these have the same domain (as $R(R(g))=R(g)$ ). Clearly, also $\xi_{R(g)}$ fixes its domain. Hence they are the same. 
Lemma 5.3. Suppose $\beta=\left(c_{0}, d_{1}, c_{1}, \ldots\right) \in W(A), g \in A$, and $\beta \lessdot L(g)$. Let $\alpha=\left(R\left(L\left(c_{0}\right) g\right), L\left(c_{0}\right) g, \beta\right)$. Then $\alpha \in \mathrm{p}(A), \alpha \lessdot g$, and $\xi_{g}(\bar{\alpha})=\beta$.

Proof. Suppose $\alpha, \beta$ are as above. Note that $\alpha$ is permissible as is easily checked. Also, $R(g) R\left(L\left(c_{0}\right) g\right)=R\left(L\left(c_{0}\right) g R(g)\right)=R\left(L\left(c_{0}\right) g\right)$ by right normality of $R$, so $\alpha \lessdot g$. Then $\xi_{g}(\bar{\alpha})=\overline{g \bar{\alpha}}=\overline{g \alpha}=\overline{\left(g R\left(L\left(c_{0}\right) g\right), L\left(c_{0}\right) g, \beta\right)}=\overline{\left(R\left(L\left(c_{0}\right)\right) g, L\left(c_{0}\right) g, \beta\right)}=$ $\overline{\left(L\left(c_{0}\right) g, L\left(c_{0}\right) g, \beta\right)}=\beta$ as is clear.

Theorem 5.4. For all $g \in A, L\left(\xi_{g}\right)=\xi_{L(g)}$.

Proof. Again, both sides are restrictions of the identity. We must show they have equal domains. Pick $\beta \in \operatorname{dom}\left(L\left(\xi_{g}\right)\right)$, so that $\xi_{g}(\alpha)=\overline{g \alpha}=\beta$ for some reduced $\alpha$, with $\alpha=\left(a_{0}, \ldots\right)$. Now $g a_{0}=L(g) g a_{0}$, so $g \alpha=L(g)(g \alpha)$, so $\overline{g \alpha}=L(g) \overline{g \alpha}$, and so $\beta=L(g) \beta=R(L(g)) \beta$, and so $\beta \lessdot L(g)$, that is, $\beta \in \operatorname{dom}\left(\xi_{L(g)}\right)$.

Conversely, suppose $\beta=\left(c_{0}, \ldots\right) \in \operatorname{dom}\left(\xi_{L(g)}\right)$, so $\beta \lessdot L(g)$. Then by Lemma 5.3 , there exists a reduced form $\alpha$ such that $\alpha \in \operatorname{dom}\left(\xi_{g}\right)$ and $\xi_{g}(\alpha)=\beta$, so $\beta \in \operatorname{ran}\left(\xi_{g}\right)$.

Theorem 5.5. $\xi$ is injective.

Proof. Let $a \neq b$ in $A$. Without loss of generality we may assume that $R(a) \leq R(b)$. If $R(a)=R(b)$ then $(R(a)) \lessdot a, b$ but $\xi_{a}((R(a)))=(a) \neq(b)=\xi_{b}((R(\bar{a})))$. If $R(a)<R(b)$ then $(R(b)) \in \operatorname{dom}\left(\xi_{b}\right) \backslash \operatorname{dom}\left(\xi_{a}\right)$.

We call the embedding just described of the type 3 function system $\mathbf{A}$ into $\mathcal{P}(A)$ its word-regular representation.

If $\mathbf{A}$ is a type 3 function system with a closed identity (so that $\mathbf{A}$ is a $\{R, L, 1\}$ semigroup), then the representation $\xi$ obviously maps 1 to the identity map on $W(A)$. If $\mathbf{A}$ has a closed zero element (so that $\mathbf{A}$ is a $\{R, L, 0\}$-semigroup), then it is not true that $\xi_{0}$ is the empty map. However the map $\xi^{b}$ provides a representation for $\mathbf{A}$ into $\mathcal{P}(W(A) \backslash\{(0)\})$ in which $\xi_{0}^{b}=\varnothing$. Indeed, the point (0) in $W(A) \backslash\{(0)\}$ is obviously fixed by every element of $\xi(A)$ but Lemma 4.2 shows that if $g \in A$ has $\xi_{g}(\alpha)=(0)$ then $\alpha=\left(a_{0}\right)$ for some $a_{0}$ with $g a_{0}=0$. As $\alpha \lessdot g$ we have $a_{0}=R(g) a_{0}=a_{0} R\left(g a_{0}\right)=a_{0} R(0)=a_{0} 0=0$. Hence a version of Lemma 3.1 holds and $\xi^{b}$ is also a representation.

The following corollary summarises the results of this section. (Aside from the simple modifications to include 0 and 1, this result is due to Schein, 1970a.)

Corollary 5.6. Let $\{R, L\} \subseteq \Lambda \subseteq\{R, L, 0,1\}$. The representable $\Lambda$-semigroups are precisely those that are type 3 function systems as $\{R, L\}$-semigroups.

\section{Special CASE: inverse SEMigroups AND injeCtive FunCtions.}

Suppose $\mathbf{A}=\left\langle A ; \cdot,{ }^{\prime}\right\rangle$ is an inverse semigroup and let $R, L$ be defined in the usual way: $L(x):=x x^{\prime}$ and $R(x):=x^{\prime} x$. Then $A$ is a type 3 function system: $\langle A, \cdot, R\rangle$ is right twisted, $\langle A, \cdot, L\rangle$ is left twisted, $R(A)=L(A)$ and if $a c=b c$ then $a c c^{\prime}=b c c^{\prime}$, that is $a L(c)=b L(c)$ (so (L6) holds).

Theorem 6.1. Let $A$ be an inverse semigroup with usual closure. Then under the word-regular representation, for each $f \in A, \xi_{f}$ is injective, and indeed $\xi_{f}^{\prime}=\left(\xi_{f}\right)^{-1}$.

Proof. For all $f \in A, \xi_{f} \circ \xi_{f^{\prime}}=\xi_{f f^{\prime}}=\xi_{L(f)}$, and similarly $\xi_{f^{\prime}} \circ \xi_{f}=\xi_{R(f)}$. Hence if $\xi_{f}(\alpha)=\beta$ for some $\alpha, \beta \in W(A)$, then $\xi_{f^{\prime}}(\beta)=\xi_{f^{\prime}} \circ \xi_{f}(\alpha)=\xi_{R(f)}(\alpha)=\alpha$, and similarly if $\xi_{f^{\prime}}(\beta)=\alpha$, then $\xi_{f}(\alpha)=\beta$. 
It would be interesting if one could extract from the word-regular representation, a method of characterising $\{L, R\}$-semigroups of injective functions. Indeed this is easy to do in the case of $\{R\}$-semigroups: only one extra quasi-equation is required. (The class obtained is precisely the right ample semigroups - also known as right type A semigroups; see Fountain, 1979.) However the following result of Gould and Kambites (2005) shows that there can be no "easy" solution to the two sided version.

Theorem 6.2. (Gould and Kambites, 2005) There is no algorithm to decide the following problem: given a finite algebra $A$ of type $\langle 2,1,1\rangle$, decide if $A$ isomorphic to an $\{L, R\}$-semigroup of injective functions.

Proof. Recall that the ample semigroups (in the type $\langle 2,1,1\rangle$ ) are the $\{L, R\}$ semigroups satisfying right twistedness of $R$ (law (R5)), left twistedness of $L$ (the left dual to law (R5)) as well as Schein's quasi-identity (L6) and its right dual

$$
x y \approx x z \rightarrow R(x) y \approx R(x) z .
$$

We can assume that $A$ is an ample semigroup, since this property is decidable and necessary for representability as an $\{L, R\}$-semigroup of injective functions. If the ample semigroup $A$ is embeddable in an inverse semigroup (as an $\{L, R\}$ semigroup), then it is representable as injective functions, since inverse semigroups are so representable. Conversely, an ample semigroup representable as injective functions on a set $X$ embeds into the symmetric inverse semigroup $I(X)$ (as an $\{L, R\}$-semigroup). Gould and Kambites (2005; Theorem 3.4 combined with Corollary 4.3) show that embeddability of finite ample semigroups in inverse semigroups (as $\{L, R\}$-semigroups) is undecidable.

It follows that there can be no axiomatisation for $\{L, R\}$-semigroups of injective functions that is algorithmically verifiable on finite algebras. In the case of the wordregular representation, it turns out that the only elements that are represented as injective functions are those that already have an inverse.

Proposition 6.3. Suppose $A$ is a type 3 function system such that $\xi_{f}$ is injective for all $f \in A$. Then $A$ is an inverse semigroup with the usual left and right closures.

Proof. Suppose $\xi_{f}$ is injective. Now $R(f)^{2}=R(f)$, so $(R(f)) \lessdot f$, and $\xi_{f}((R(f))=$ $(f)$. (All words here are trivially permissible and reduced!) But by Lemma 5.3, if $\alpha=(R(L(f) f), L(f) f, f)=(R(f), f, f)$, then $\bar{\alpha}$ maps to $(f)$ also, so by injectivity, $\bar{\alpha}=(R(f))$. So $(R(f), f, f)$ reduces to $(R(f))$, and so $R(f)=x f$ for some $x \in A$.

The result now follows from Theorem 3.6 of Jackson and Stokes (2001) and the fact that an idempotent injective partial map is necessarily a restriction of the identity map (hence a fixed point of $R$ and $L$ ). We give an independent proof here for completeness.

Now $R(x) f=f$ and then $\xi_{x}((f))=(x f)$. But also, $(R(L(x f) x), L(x f) x, x f)$ maps to $(x f)$ by Lemma 5.3 , so $R(L(x f) x)=y L(x f) x$ for some $y$, and $f=R(x) f=$ $y x f=y R(f)$, so $f \leq y$.

But $L(x f)=L(R(f))=R(f)$, and then $R(f x)=R(R(f) x)=y R(f) x=f x$, so $f x$ is closed. Moreover $(f x) f=f(x f)=f R(f)=f$, so $f x \geq L(f)$. Also, $L(f) L(f x)=L(f x)=f x$, so $f x=L(f)$.

Hence $x f=R(f), f x=L(f)$, and so an argument as in the proof of the Theorem 6.1 establishes that $\xi_{x}=\left(\xi_{f}\right)^{-1}$. So all of $\mathbf{A}$ is represented as injective partial 
maps together with their inverses, and $\mathbf{A}$ must be inverse (and $R, L$ are the usual closures).

\section{Representing TWo-Sided AGREEABle SEMigroups}

The algebra $\langle\mathcal{P}(X) ; \circ, R, L, \wedge\rangle$ is a twisted SLORC as well as a type 3 function system. We now show that the word-regular representation extends to $\wedge$ if it is twisted, thereby giving an abstract characterisation of subalgebras of $\langle\mathcal{P}(X) ; \circ, \wedge, R, L\rangle$. We prove this in terms of the associated right agreeable operation $*$.

Lemma 7.1. Suppose $\mathbf{A}=\langle A ; \cdot, *\rangle$ is a twisted right agreeable semigroup. Then for all $f, g, h \in A, f=(g * h) f$ if and only if $g f=h f$ and $R(g) f=R(h) f$.

Proof. If $f=(g * h) f$ then $g f=g(g * h) f=h(g * h) f=h f, R(g) f=R(g)(g * h) f=$ $(g * h) f=f$, and similarly $R(h) f=f$. Conversely, if $g f=h f$ and $R(g) f=R(h) f$ then $(g * h) f=f(g f * h f)=f(g f * g f)=f R(g f)=R(g) f=f$.

Theorem 7.2. Suppose $\mathbf{A}=\langle A ; \cdot, *, L\rangle$ is a twisted right agreeable semigroup, with the operation $R(x)=x * x$ making $\langle A ; \cdot, R, L\rangle$ a type 3 function system. Then for all $f, g \in A$ we have $\xi_{g} * \xi_{h}=\xi_{g * h}$.

Proof. For $\alpha=\left(a_{0}, \ldots\right) \in W(A), \alpha \in \operatorname{dom}\left(\xi_{g} * \xi_{h}\right)$ if and only if $\alpha \in \operatorname{dom}\left(\xi_{g}\right)$ and $\alpha \in \operatorname{dom}\left(\xi_{h}\right)$ and $\xi_{g}(\alpha)=\xi_{h}(\alpha)$, if and only if $\alpha \lessdot g, h$ and $\overline{g \alpha}=\overline{h \alpha}$, if and only if $\alpha \lessdot g, h$ and $g \alpha=h \alpha$ by Lemma 4.6, if and only if $R(g) a_{0}=a_{0}=R(h) a_{0}$ and $g a_{0}=$ $h a_{0}$, if and only if $(g * h) a_{0}=a_{0}$ by Lemma 7.1, if and only if $\alpha \in \operatorname{dom}\left(\xi_{g * h}\right)$.

Hence the twisted right agreeable type 3 function system $\mathbf{A}$ is embeddable in $\langle\mathcal{P}(W(A)) ; \circ, *, L\rangle$. In particular this means that the meet operation on $\mathbf{A}$ (given by $a \wedge b=a R(a * b))$ is represented as intersection of partial maps.

Corollary 7.3. The representable $\{R, L, \wedge\}$-semigroups are the type 3 function systems that are twisted SLORCs.

This extends both the abstract characterisation of subalgebras of $\langle\mathcal{P}(X) ; \circ, R, L\rangle$ in Schein (1970a) and of subalgebras of $\langle\mathcal{P}(X) ; \circ, \cap, R\rangle$ in Dudek and Trokhimenko (2002) and Jackson and Stokes (2003).

Next we show that if a type 3 function system is left agreeable, say $\mathbf{A}=\langle A ; \cdot, \Lambda\rangle$ where $\{R, \star\} \leq \Lambda$, then the word-regular representation extends to the left agreeable operation. It turns out that no conditions are required other than the derived $R$ and $L$ making $\langle S ; \cdot, R, L\rangle$ a type 3 function system.

Theorem 7.4. Suppose $\mathbf{A}=\langle A ; \star, R\rangle$ is left agreeable semigroup with the operation $L(x)=x \star x$ making $\langle A ; \cdot, R, L\rangle$ a type 3 function system. Then for all $f, g \in A$ we have $\xi_{f \star g}=\xi_{f} \star \xi_{g}$.

Proof. Each side of the above equality is a restriction of the identity - we must show their domains are equal. Because $L$ satisfies the left congruence condition (L5), the (left) "normality law" $\alpha(x \star y) \approx(\alpha x \star y)$ (for $\alpha$ any closed element) holds; see Proposition 5.4 of Jackson and Stokes (2003). By Theorem 5.1 of Jackson and Stokes (2003), this in turn shows that

$$
(f \star g)=\max \{e \in R(A) \mid e f=e g, e \leq L(f), L(g)\} .
$$

The properties $e f=e g$ and $e \leq L(f), L(g)$ (for $e \in R(A)$ ) are defined in terms of $\cdot, R, L$ which are preserved by $\xi$ and so we immediately obtain $\operatorname{dom}\left(\xi_{f \star g}\right) \subseteq$ $\operatorname{dom}\left(\xi_{f} \star \xi_{g}\right)$. 
Conversely, suppose $\beta \in \operatorname{dom}\left(\xi_{f} \star \xi_{g}\right)$, that is, $\beta \in \operatorname{ran}\left(\xi_{f}\right) \cap \operatorname{ran}\left(\xi_{g}\right)$, and $\xi_{f}(\alpha)=$ $\beta$ if and only if $\xi_{g}(\alpha)=\beta$. Say $\beta=\left(c_{0}, \ldots\right)$. So $\beta \lessdot L(f), L(g)$ by Theorem 5.4. So by Lemma 5.3 , letting $\alpha=\left(R\left(L\left(c_{0}\right) f\right), L\left(c_{0}\right) f, \beta\right)$, where $\beta=\left(c_{0}, \ldots\right)$, we have that $\alpha$ is permissible, $\alpha \lessdot f$, and $\xi_{f}(\bar{\alpha})=\beta$, so it must be that $\alpha \lessdot g$ and $\xi_{g}(\bar{\alpha})=\beta$ also. Hence $\overline{f \bar{\alpha}}=\overline{g \bar{\alpha}}$, and so $f \alpha=g \alpha$ by Corollary 4.8 , so $L\left(c_{0}\right) f=g R\left(L\left(c_{0}\right) f\right)$, so $L\left(c_{0}\right) f \leq_{R} g$, so $L\left(c_{0}\right) f \leq_{R} L\left(c_{0}\right) g$. Hence by symmetry we have $L\left(c_{0}\right) f=L\left(c_{0}\right) g$. But $\beta \lessdot L(f), L(g)$, so $L(f) c_{0}=c_{0}=L(g) c_{0}$, so $L(f), L(g) \geq L\left(c_{0}\right)$. This together with the fact that $L\left(c_{0}\right) f=L\left(c_{0}\right) g$ shows that

$$
L\left(c_{0}\right) \leq \max \{e \in R(A) \mid e f=e g, e \leq L(f), L(g)\}=(f \star g),
$$

and so $(f \star g) c_{0}=c_{0}$. Therefore $\beta \lessdot(f \star g)$; that is, $\beta \in \operatorname{dom}\left(\xi_{f \star g}\right)$.

Corollary 7.5. The representable $\{R, L, \curlywedge\}$-semigroups are the type 3 function systems with $\curlywedge$.

If a closed 0 is present, then the results in this section are also trivially seen to hold using the representation $\xi^{b}$. As an immediate consequence of Theorems 7.2 and 7.4 is the following.

Corollary 7.6. Let $\{R, L\} \leq \Lambda \leq\{R, L, \wedge, \curlywedge, *, \star, 0,1\}$. A $\Lambda$-semigroup is representable if and only if it is a type 3 function system with respect to $R$ and $L$ and twisted with respect to $\wedge$ or $*$ (if they are present).

A two sided agreeable semigroup (possibly with a closed 0 and/or a closed 1 ) is representable if and only if it is right twisted and a type 3 function system with respect to the derived operations $R$ and $L$.

Our axiomatisations of these classes all involve the quasi-identity (L6): $x z \approx$ $y z \rightarrow x L(z) \approx y L(z)$. In Section 9 we show that this implication is equivalent to an identity for some of the above choices of $\Lambda$, in particular, when any one of $\wedge$, $\curlywedge$ (and therefore $*$ or $\star$ ) are present. So in contrast with type 3 function systems, representable two sided agreeable semigroups form a variety. Moreover, most of the known examples of algebras representable in terms of partial maps are reducts of this variety (see Schein, 1970b, and Jackson and Stokes, 2007?, for example).

\section{INTERIOR OPERATIONS}

There is a number of other operations that lie in between $\{R, L\}$ and $\{R, L, \wedge, \curlywedge\}$ in their expressive strength. In particular, we have seen that the operations $I$ and $J$ are expressible as the terms $x \wedge R(x)$ and $x \curlywedge L(x)$ respectively (Lemma 2.3). As a consequence, these operations are trivially seen to be preserved by Schein's representation $\xi$ if the more powerful operations $\wedge$ and $\curlywedge$ are present. If $\wedge$ and $\curlywedge$ are not present, then it is not immediately clear that $I$ and $J$ are preserved. For example, the problem of describing the class of representable $\{R, L, I, J\}$-semigroups is equivalent to describing the class of $\{R, L, I, J\}$-semigroups embeddable in the $\{\cdot, R, L, I, J\}$-reduct of a representable two sided agreeable semigroup. In this section we show that this problem has an easy solution.

Recall that an $\{R, I\}$-semigroup is representable if and only if it is representable as an $R$ semigroup and the interior operation $I$ satisfies the implication (I5): $a b \approx b \rightarrow I(a) b \approx b$. We show that this carries over to the situation where some combination of $L, J$, and $\curlywedge$ are present. 
Theorem 8.1. Let $\Lambda$ be a well-formed subset of $\{R, L, I, J, \wedge, \curlywedge, 0,1\}$ containing $R, L, I$, and let $\mathbf{S}$ be a $\Lambda$-semigroup for which $\langle S ; R, L\rangle$ is a type 3 function system. The representation $\xi$ preserves $I$ if and only if (I5) is satisfied.

Proof. This is an easy modification of the proof of Theorem 3.4 using the word regular representation in place of the standard RC-semigroup representation.

There is another path to this result that leads to an equivalent but different axiomatisation. If $*$ is present, then the value $I(x)$ is equal to $x * R(x)$. This suggests that it might be possible to replicate the proofs of Lemma 7.1 and Theorem 7.2 , replacing $h$ everywhere by $R(g)$. Each time a property of $*$ is used in these proofs, we hope to find a way of expressing the corresponding property for $I$. This is easy if each expression $(a * b)$ in the proof has $\{a, b\}$ equal to $\{c, R(c)\}$ for some $c$ (whence $a * b=I(c)$ ), but problems may arise otherwise. For example, in the final line of the translated proof of Lemma 7.1 there would appear $(g * R(g)) f=$ $f(g f * R(g) f)=f(g f * g f)=f R(g f)=R(g) f=f$, which holds because the earlier part of the proof shows that $g f=R(g) f$. It is not clear how to write the expression $g f * R(g) f$ in terms of $I$, but to circumvent this, we can include the implication $g f \approx R(g) f \rightarrow I(g) f \approx f R(g f)$ in our axioms. The implications one is forced to include are certainly necessary for representability - because they correspond to implications in $*$ that we deduced for representable agreeable semigroups - but they are also sufficient, because together they give the proof of the translated versions of Lemma 7.1 and Theorem 7.2.

Let us apply this idea to the left interior operation $J$. In this case it turns out that no extra laws are required.

Theorem 8.2. Let $\{R, L, J\} \leq \Lambda \leq\{R, L, I, J, \wedge, \curlywedge, 0,1\}$ and $\mathbf{S}$ be a $\Lambda$-semigroup for which $\langle S ; R, L\rangle$ is a type 3 function system. The representation $\xi$ preserves $J$ if and only if $\langle S ; \cdot, R, L, J\rangle$ is an $\{R, L, J\}$-semigroup.

Proof. Our aim is to follow through the proof of Theorem 7.4, using $L(f)$ in place of $g$. By looking at the proof it is clear that the necessary and sufficient property for representability is that $J(x)=\max \{e \in R(S) \mid e x=e L(x) \& e \leq L(x)\}$. This is easily axiomatised by the laws $J(x) \approx R(J(x)), J(x) x \approx J(x) L(x), J(x) \leq L(x)$ and $y \approx R(y) \& y x \approx y L(x) \& y \leq L(x) \rightarrow y \leq_{R} J(x)$. We now claim that these properties hold provided only that $J$ is a left interior operation mapping onto the closed elements.

Certainly, if $\langle S ; R, L, J\rangle$ is representable then it is a $\{R, L, I\}$-semigroup for which $\langle S ; R, L\rangle$ is a type 3 function system. Now assume that $\langle S ; R, L, J\rangle$ is an $\{R, L, I\}$-semigroup with $\langle S ; R, L\rangle$ a type 3 function semigroup. So the identity $J(x) \approx R(J(x))$ holds and $J(x) x \approx J(x) L(x) x \approx L(x) J(x) \approx L(x) J(x)$. Also,

$$
J(x) L(x) \approx L(J(x) L(x)) \approx L(J(x) x) \approx L(J(x)) \approx J(x) .
$$

This shows that for any $a \in S$ we have $J(a) \in\{e \in R(S) \mid a x=a L(a)$ \& $e \leq$ $L(a)\}$. Finally, say that $e$ is a closed element and $a \in S$ is such that $e a=e L(a)$ and $e \leq L(a)$. Then $e=e L(a)=e a$, so $e \leq J(a)$ as required.

Both Theorems 8.1 and 8.2 hold if a closed 0 or 1 are present (using $\xi^{b}$ in place of $\xi$ if 0 is present).

It should be fairly clear that one can replicate these ideas for many other term operations in the language $\cdot R, L, *, \star$, thereby characterising the representable algebras. For example, the proof of Theorem 8.2 is easily adapted to accommodate 


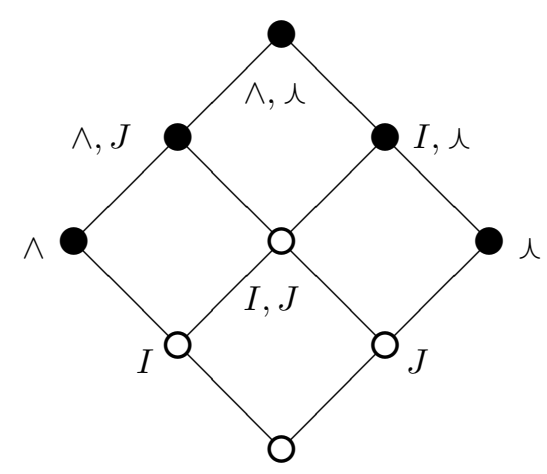

FiguRE 1 . The representable $\Lambda$-semigroups: varieties versus quasivarieties.

the operation $x \curlywedge R(x)$ (which is also equal to $J(x) R(x)$, if $J$ is present). However, except in a few cases such as $I$ and $J$ the actual definitions of these operations are not particularly natural, so we do not here attempt to pursue a general approach to axiomatising them. A similar situation occurs in a companion paper, Jackson and Stokes (2006b), where representations are described for partial maps on a quasi-ordered set. The definition of the operations $R, L, *, \star$ are altered to describe natural properties of the underlying quasi-order (so the representation and the represented objects are quite distinct from those considered in this article), however the collection of operations that can be represented is extended to include some of much greater expressive power than $*$ and $\star$ in the hierarchy of semilattice valued operations. Accordingly, there are a greater number of derived operations and it becomes of more interest to take the general approach alluded to here.

\section{VARIETIES}

The definition of a type 3 function system involves a quasi-identity and Schein showed that there is no equivalent equational definition. This quasi-identity (and one other) percolates throughout our extensions of Schein's result, however it turns out that in many cases it can be replaced by an equation.

Theorem 9.1. Let $\{R, L\} \leq \Lambda \leq\{R, L, I, J, \wedge, \curlywedge, 0,1\}$. The class of representable $\Lambda$-semigroups forms a variety if and only if $\{\Lambda\} \leq \Lambda$ or $\{\curlywedge\} \leq \Lambda$. In particular, the class of representable two sided agreeable semigroups is a variety.

Theorem 9.1 is summarised in Figure 1. The diagram describes which of the 9 possible distinct choices of $\Lambda$ satisfying $\{R, L\} \leq \Lambda \leq\{R, L, I, J, \wedge, \curlywedge\}$ are varieties and which are quasivarieties (the presence of 0 and 1 is omitted since we have already shown these can be treated equationally). The operations $\cdot R, L$ are assumed throughout and are omitted from the labels. The order in the diagram represents the relative expressive power of the various operations. So the vertex labelled $\wedge$ is ordered above that labelled $I$ because every $\{R, L, \wedge\}$-semigroup is an $\{R, L, I\}$ semigroup (as $I(x)=x \wedge R(x)$ ). The solid vertices indicate that the corresponding class of representable algebras forms a variety, while the hollow vertices correspond to proper quasivarieties. 
Note that we have also observed characterisations for the representable $\{R\}$-, $\{R, I\}$ - and $\{R, \wedge\}$-semigroups but these are not included in Theorem 9.1. The representable $\{R\}$ - and $\{R, \wedge\}$-semigroups are the varieties of twisted RC-semigroups and twisted SLORCs, respectively, while Lemma 9.3 below will show that the representable $\{R, I\}$-semigroups form a proper quasivariety. We also observed that the algebras representable as subalgebras of $\langle\mathcal{P}(X) ; \circ, \wedge\rangle$ were characterised by Garvac'kil (1971) who showed that they are a finitely based variety.

9.1. Proof of Theorem 9.1. Theorem 9.1 is proved over a series of lemmas. We begin by proving that if $\wedge$ and $\curlywedge$ are not present, then the corresponding class is not closed under homomorphic images (Lemmas 9.2 and 9.3). Now recall that the laws characterising representability in the above section are identities except for the following two cases:

(L6) $a c \approx b c \rightarrow a L(c) \approx b L(c) ;$

(I5) $a b \approx b \rightarrow I(a) b \approx b$.

To complete the proof of Theorem 9.1 it will then suffice to show that if either of $\wedge$ or $\curlywedge$ are present, then (L6) and (I5) are equivalent to identities (Lemmas 9.5-9.8).

Lemma 9.2. Let $\Lambda$ be any subset of $\{R, L, I, J, 0,1\}$ containing $L$. Then the class of representable $\Lambda$-semigroups is a proper quasivariety.

Proof. We construct a representable $\{R, L, I, J, 0,1\}$-semigroup $\mathbf{S}$ that has a quotient failing quasi-identity (L6) and hence is not representable. Hence the $\Lambda$ semigroup reduct of $\mathbf{S}$ is representable but has a non-representable quotient.

Each partial map $f: X \rightarrow X$ can be described by a matrix with 2 rows and a column for each element in the domain of $f$. The first row contains - without repeats - the elements in the domain of $f$. The second row contains the corresponding values of $f$. We use this notation here and elsewhere below.

Let $\mathbf{6}$ be the six element set $\{0,1,2,3,4,5\}$ and let $\mathbf{S}$ be the subalgebra of the concrete algebra $\langle\mathcal{P}(\mathbf{6}) ; \circ, R, L, I, J, \varnothing, \mathrm{id}\rangle$ consisting of the (injective) partial maps

$$
\begin{gathered}
a:=\left(\begin{array}{ll}
2 & 3 \\
4 & 5
\end{array}\right), \quad b:=\left(\begin{array}{ll}
2 & 3 \\
5 & 4
\end{array}\right), \quad c:=\left(\begin{array}{ll}
0 & 1 \\
2 & 3
\end{array}\right), \quad a c=\left(\begin{array}{ll}
0 & 1 \\
4 & 5
\end{array}\right), \quad b c=\left(\begin{array}{ll}
0 & 1 \\
5 & 4
\end{array}\right), \\
\left(\begin{array}{ll}
0 & 1 \\
0 & 1
\end{array}\right), \quad\left(\begin{array}{ll}
2 & 3 \\
2 & 3
\end{array}\right), \quad\left(\begin{array}{ll}
4 & 5 \\
4 & 5
\end{array}\right),
\end{gathered}
$$

along with the identity $\left(\begin{array}{llllll}0 & 1 & 2 & 3 & 4 & 5 \\ 0 & 1 & 2 & 3 & 4 & 5\end{array}\right)$ and the empty map. Note that $L(c)$ is $\left(\begin{array}{ll}2 & 3 \\ 2 & 3\end{array}\right)$ so that $a L(c)=a$ and $b L(c)=b$. It is easily verified that the equivalence $\theta$ identifying the elements $a b$ and $a c$ but no other elements is a congruence. As $a / \theta \cdot L(c) / \theta=a / \theta=\{a\} \neq\{b\}=b / \theta \cdot L(c) / \theta$, the quasi-identity (L6) fails and $\mathbf{S} / \theta$ is not representable.

The case $\Lambda=\{R, L\}$ was shown to give rise to a proper quasivariety by Schein (1970a) using an infinite example.

Lemma 9.3. Let $\Lambda$ be any subset of $\{R, L, I, J, 0,1\}$ containing I. Then the class of representable $\Lambda$-semigroups is a proper quasivariety. 
Proof. The proof method is similar to that of Lemma 9.2. Let $\mathbf{T}$ be the $\{R, L, I, J, 0,1\}$ semigroup consisting of the following (injective) partial maps on the four element set $\mathbf{4}=\{0,1,2,3\}$ :

$$
a:=\left(\begin{array}{ll}
0 & 1 \\
1 & 0
\end{array}\right), \quad\left(\begin{array}{ll}
0 & 1 \\
0 & 1
\end{array}\right), \quad b:=\left(\begin{array}{ll}
2 & 3 \\
0 & 1
\end{array}\right), \quad a b=\left(\begin{array}{ll}
2 & 3 \\
1 & 0
\end{array}\right), \quad\left(\begin{array}{ll}
2 & 3 \\
2 & 3
\end{array}\right)
$$

along with the identity and empty maps. It is easily verified that the equivalence $\rho$ identifying $a b$ and $b$ is a congruence. But then in $\mathbf{S}$ we have $a / \rho \cdot b / \rho=b / \rho$, while $I(a)=\varnothing$ so that $I(a) / \rho \cdot b / \rho \neq b / \rho$ showing that $\mathbf{S} / \rho$ is not representable.

Surprisingly, the quasi-identity (I5) is actually equational in any finite aperiodic semigroup. Indeed, if $x^{\omega}$ denotes the unique idempotent power of an element $x$ in a finite semigroup, then we have the following.

Lemma 9.4. Let $\Lambda$ be a subset of $\{R, L, I, J, \wedge, \curlywedge, 0,1\}$ containing $I$ and let $\mathbf{S}$ be a finite $\Lambda$-semigroup whose subgroups are trivial. Then $\mathbf{S}$ satisfies the implication (I5) if and only if it satisfies $I\left(x^{\omega}\right) x^{\omega} \approx x^{\omega}$.

Proof. Certainly if (I5) holds, then as $x^{\omega} x^{\omega}=x^{\omega}$ we have $I\left(x^{\omega}\right) x^{\omega}=x^{\omega}$. Now suppose $I\left(x^{\omega}\right) x^{\omega}=x^{\omega}$ for every $x \in S$ and that $\mathbf{S}$ has only trivial subgroups. In particular we have $x^{\omega}=x^{\omega} x$. Now $I(x) x^{n}=I(x)$ for any $n$, so $I(x) \leq I\left(x^{\omega}\right)$. However $I\left(x^{\omega}\right) x=I\left(x^{\omega}\right) x^{\omega} x=I\left(x^{\omega}\right) x^{\omega}=I\left(x^{\omega}\right)$, so that $I\left(x^{\omega}\right) \leq I(x)$ as well. Hence $I(x)=I\left(x^{\omega}\right)$. Suppose $a b=b$ in $\mathbf{S}$. Then $a^{\omega} b=b$ and so $I(a) b=I\left(a^{\omega}\right) b=$ $I\left(a^{\omega}\right) a^{\omega} b=a^{\omega} b=b$.

It follows, for example, that while the class of finite representable $\{R, I\}$-semigroups is not closed under homomorphic images, the class of finite aperiodic representable $\{R, I\}$-semigroups is closed under taking subalgebras, homomorphic images and finitary direct products; that is, is a pseudovariety. We now show that in all other cases of $\{R, L\} \leq \Lambda \leq\{R, L, I, J, \wedge, \curlywedge, 0,1\}$, the corresponding class is a variety.

Lemma 9.5. Consider $\{R, L, I, \curlywedge\} \leq \Lambda \leq\{R, L, I, J, \wedge, \curlywedge, 0,1\}$ and let $\mathbf{S}$ be a $\Lambda$-semigroup that is a type 3 function system with respect to $R, L$. Then the quasiidentity (I5) is equivalent to the law

$$
I(x)(x y \curlywedge y) \approx x y \curlywedge y .
$$

Proof. Let $\mathbf{S}$ be a $\{R, L, I, \curlywedge\}$-semigroup that is a type 3 function system with respect to $R, L$. The inequality $I(a)(a b \curlywedge b) \leq_{R} a b \curlywedge b$ holds for any $a, b \in S$ since $I(a)(a b \curlywedge b)=R(I(a))(a b \curlywedge b)=(a b \curlywedge b) R(I(a)(a b \curlywedge b))$ (using (R5)).

Now say that (I5) holds on $\mathbf{S}$. By Theorem 8.1, we may assume that $\mathbf{S}$ is an $\{R, L, I, \curlywedge\}$-semigroup of partial maps on a set $X$. Consider any $a, b \in S$. Say that $[a b \curlywedge b](i)=j$ for some $i, j \in X$. So $[a b](i)=j$ and $[b](i)=j$. Hence $[a](j)=$ $[a]([b](i))=[a b](i)=j$. Hence $[I(a)](j)=j$ also, and so $[I(a)(a b \curlywedge b)](i)=j$. This shows that the revese inequality $a b \curlywedge b \leq_{R} I(a)(a b \curlywedge b)$ holds and so identity (I $5^{\curlywedge}$ ) is satisfied.

Conversely, say that identity ( $\left(5^{\curlywedge}\right)$ holds on $\mathbf{S}$. Say that $a b=b$ for some $a, b \in S$. Then $a b \curlywedge b=b$ showing that $I(a) b=I(a)(a b \curlywedge b)=a b \curlywedge b=b$, showing that (I5) holds.

The operation $I$ can be written in terms operations $R, \wedge$ in the representable case: namely $I(x):=x \wedge R(x)$. Since the representable $\{R, \wedge\}$-semigroups form a variety we obtain the following lemma. 
Lemma 9.6. Consider $\{R, I, \wedge\} \leq \Lambda \leq\{R, L, I, J, \wedge, \curlywedge, 0,1\}$ and let $\mathbf{S}$ be a $\Lambda$ semigroup that is a twisted $S L O R C$ with respect to $\{R, \wedge\}$. Then the quasi-identity (I5) is equivalent to the law

$$
I(x) \approx x \wedge R(x) .
$$

It remains to show that implication (L6) can be made equational in the operation $\curlywedge$ and also in the operation $\wedge$.

Lemma 9.7. Let $\Lambda$ be a subset of $\{R, L, I, J, \wedge, \curlywedge, 0,1\}$ containing $L$, $\curlywedge$ and let $\mathbf{S}$ be a $\Lambda$-semigroup satisfying the left congruence condition on $L$. Then the quasiidentity (L6) is equivalent to the law

$$
a c L(a c \curlywedge b c) \approx a L(c)(a L(c) \curlywedge b L(c)) .
$$

Proof. We use the more succinct $\star$ notation so the identity becomes $a c \star b c \approx$ $a L(c) \star b L(c)$.

Suppose (L6 $\left.6^{\curlywedge}\right)$ holds. If $a, b, c \in S$ are such that $a c=b c$, then (L6 $\left.6^{\curlywedge}\right)$ implies $(a c \star b c)=(a c \star b c)(a L(c) \star b L(c))$. We get

$$
\begin{aligned}
a L(c) & =L(a L(c)) a L(c) \\
& =L(a c) a L(c) \quad(b y(\mathrm{~L} 5)) \\
& =(a c \star a c) a L(c) \\
& =(a c \star b c) a L(c) \\
& =(a c \star b c)(a L(c) \star b L(c)) a L(c) .
\end{aligned}
$$

By swapping $a, b$ we get $b L(c)=(a c \star b c)(a L(c) \star b L(c)) b L(c)$ which in turn is $(a c \star b c)(a L(c) \star b L(c)) a L(c)=a L(c)$.

Conversely, suppose the implication holds. Now for every $a, b, c \in S$ we have $(a c \star b c) a c=(a c \star b c) b c$, so $(a c \star b c) a L(c)=(a c \star b c) b L(c)$. Moreover, $(a c \star$ $b c) L(a L(c)) L(b L(c))=(a c \star b c) L(a c) L(b c)=(L(a c) a c \star L(b c) b c)=(a c \star b c)$, so $(a c \star b c) \leq L(a L(c)) L(b L(c))$. This together with the fact that $(a c \star b c) a L(c)=$ $(a c \star b c) b L(c)$ and the left normality of $L$ imply that $(a c \star b c) \leq(a L(c) \star b L(c))$.

For the reverse inequality we have

$$
\begin{aligned}
(a c \star b c)(a L(c) \star b L(c)) & =(a L(c) c \star b L(c) c)(a L(c) \star b L(c)) \\
& =(b L(c) c \star b L(c) c)(a L(c) \star b L(c)) \\
& =(b c \star b c)(a L(c) \star b L(c)) \\
& =L(b c)(a L(c) \star b L(c)) \\
& =L(b L(c))(a L(c) \star b L(c)) \\
& =(a L(c) \star L(b L(c)) b L(c)) \quad(b y(L 5)) \\
& =(a L(c) \star b L(c)),
\end{aligned}
$$

and so $(a L(c) \star b L(c)) \leq(a c \star b c)$.

Lemma 9.8. Let $\Lambda$ be a subset of $\{R, L, I, J, \wedge, \curlywedge, 0,1\}$ containing $R, L, \wedge$ and let $\mathbf{S}$ be a $\Lambda$-semigroup satisfying the left congruence condition on $L$ and that is twisted as a SLORC. Then the quasi-identity (L6) holds. 
Proof. Suppose $a c=b c$ in $\mathbf{S}$. Then

$$
\begin{aligned}
R(a) L(c) & =L(R(a) L(c)) \\
& =L(R(a) c) \quad(\text { by }(\mathrm{L} 5)) \\
& =L(c R(a c)) \quad(\text { by }(\mathrm{R} 5)) \\
& =L(c(a c * a c)) \\
& =L(c(a c * b c)) \\
& =L((a * b) c) \quad(\text { by twistedness of } *) \\
& =L((a * b) L(c)) \quad(\text { by }(\mathrm{L} 5)) \\
& =(a * b) L(c)
\end{aligned}
$$

and likewise $R(b) L(c)=(a * b) L(c)$. So $a L(c)=a R(a) L(c)=a(a * b) L(c)=$ $b(a * b) L(c)=b R(b) L(c)=b L(c)$ as required.

\section{Questions.}

To complete this article we paraphrase two questions from Jackson and Stokes (2007?) (where some of the main results of this article were announced).

Question. (Jackson and Stokes, 2007?, Question 13.4.) Is every finite type 3 function system finitely representable (that is, representable as partial maps on a finite set)?

The same could be asked for two sided agreeables.

Question. (Jackson and Stokes, 2007?, Question 14.2.) Is the equational theory of the representable two-sided agreeable semigroups decidable? If so, is there a good algorithm for it?

\section{REFERENCES}

Batbedat, A. (1981). $\gamma$-demi-groups, demi-modules, produit demi-directs Semigroups, Proceedings, Oberwolfalch, Germany 1978, Lecture Notes in Mathematics 855, Springer-Verlag, $1-18$.

Bredihin, D.A. (1976). Inverse semigroups of local automorphisms of universal algebras, Sibirsk. Mat. Ž. 17:499-507, 715 [Russian; English translation in Siberian Math. J. 17 (1976), no. 3:386-392].

Bredihin D.A. (1977). An abstract characterization of some classes of algebras of binary relations, Algebra and Number Theory, No. 2, pp. 3-19. Kabardino-Balkarsk. Gos. Univ., Nalchik, 1977 [Russian].

Cockett, J.R.B. and Lack, S. (2002). Restriction categories I: categories of partial maps, Theoret. Comput. Sci. 270:223-259.

Desharnais, J., Möller, B., Struth, G. (2006). Kleene algebra with domain, ACM Trans. Comput. Log. 7:798-833.

Dudek, W., Trokhimenko, V.S. (2002). Functional Menger P-algebras, Comm. Algebra 30:5921-5931.

Fountain, J. (1979). Adequate semigroups, Proc. Edinburgh Math. Soc. 22:113-125.

Fountain, J. (1991). Free right type A semigroups, Glasgow Math. J. 33:135-148.

V.S. Garvac'kiŭ (1971). ก-semigroups of transformations, Teor. Polugrupp Prilozh. 2:2-13 [Russian].

Gomes, G.M.S., Gould, V. (1999). Proper weakly left ample semigroups, Internat. J. Algebra Comput. 9:721-739.

Gould, V., Kambites, M. (2005). Faithful functors from cancellative categories to cancellative monoids with an application to abundant semigroups, Internat. J. Algebra Comput. 15:683698. 
Hirsch, R., Hodkinson, I. (2001). Representability is not decidable for finite relation algebras, Trans. Amer. Math. Soc. 353:1403-1425. Zbl 0965.03079

Jackson M., Stokes, T. (2001). An invitation to C-semigroups, Semigroup Forum 62:279-310. Jackson M., Stokes, T. (2003). Agreeable semigroups, J. Algebra 266:393-417.

Jackson M., Stokes, T. (2006a). Identities in the algebra of partial maps, Internat. J. Algebra Comput. 16:1131-1159.

Jackson M., Stokes, T. (2006b), The abstract algebra of order preserving partial maps, manuscript.

Jackson M., Stokes, T. (2007?). Algebras of partial maps, to appear in Proceedings of the Special Interest Meeting on Semigroups and Related Mathematics, University of Sydney 2005. Kelarev, A., Stokes, T. (1999). Interior algebras and varieties, J. Algebra 221:50-59.

Kozen, D. (2000), On Hoare logic and Kleene algebra with tests, ACM Trans. Comput. Log. 1:60-76.

Lawson, M. (1991). Semigroups and ordered categories. I. The reduced case., J. Algebra 141:422-462.

Leech, J. (1995). Inverse monoids with a natural semilattice ordering, Proc. London Math. Soc. 70:146-182.

Manes, E. (2006). Guarded and Banded Semigroups, Semigroup Forum 72:94-120.

Menger, K. (1959). An axiomatic theory of functions and fluents, Studies Logic Found. Math., Axiomatic Method:454-473.

Schein, B.M. (1964). The representation of ordered semigroups, Mat. Sb. (N.S.) 65 (107):188197 [Russian].

Schein, B.M. (1970a), Restrictively multiplicative algebras of transformations, Izv. Vyš̌. Učebn. Zaved. Matematika 1970 no. 4 (95):91-102 [Russian].

Schein, B.M. (1970b). Relation algebras and function semigroups, Semigroup Forum 1:1-62. Schein, B.M. (1979). Lectures on semigroups of transformations, Amer. Math. Soc. Translat. 113(2):123-181.

Schein, B.M. (1992). Difference semigroups, Comm. Algebra 20:2153-2169.

Schein, B.M., Trokhimenko, V.S. (1979). Algebras of multiplace functions, Semigroup Forum $17: 1-64$.

Schweizer, B., Sklar, A. (1960). The algebra of functions, Math. Ann. 139:366-382.

Schweizer, B., Sklar, A. (1961). The algebra of functions II, Math. Ann. 143:440-447.

Schweizer, B., Sklar, A. (1965). The algebra of functions III, Math. Ann. 161:171-196.

Schweizer, B., Sklar, A. (1967). Function systems, Math. Ann. 172:1-16.

Trokhimenko, V.S. (1973). Menger's function systems, Izv. Vysš. Učebn. Zaved. Matematika, no. 11(138):71-78 [Russian].

Department of Mathematics, La Trobe University, Victoria 3086, Australia

E-mail address: M.G.Jackson@latrobe.edu.au

Department of Mathematics, University of Waikato, Private Bag 3105, Hamilton, NeW ZEALAND

E-mail address: stokes@math.waikato.ac.nz 